\title{
The design of Mechanical automatic processing equipment for refractory brick
}

\author{
LIU Ben-xue ${ }^{1}$, GUO Pei-dong ${ }^{2}$, Liu Ya-bin ${ }^{3}$ \\ ${ }^{1}$ School of Mechanical Engineering, Zhengzhou University, Zhengzhou, China \\ ${ }^{2}$ School of Mechanical Engineering, Zhengzhou University, Zhengzhou, China \\ ${ }^{3}$ School of Mechanical Engineering, Zhengzhou University, Zhengzhou, China
}

Key Words: mechanical; processing equipment for refractory brick; key mechanical structures Abstract: The shortcomings of the traditional refractory brick processing equipment with sensor and cylinder are ineffective and the high failure rate. Aiming at this situation, the mechanical automatic processing equipment without sensor and cylinder has been designed based on some software like SOLIDWORKS and CAXA. The daily processing capacity has been significantly increased with the equipment. This article mainly introduces the key structures and analyzes the operating principles of the designed equipment.

\section{Introduction}

The last procedure of brick production is firing. A series of physical and chemical reactions has been occurred during the firing process of refractory brick. The porosity of refractory brick has reduced and the volume density of brick has become bigger with these changes, so the shape of refractory brick has irregularly changed. Now, with the developing of the Iron and Steel Industry, concrete, electronic industry, glass and non-ferrous metal industry, it also puts forward higher request to the external surface quality of the refractory brick ${ }^{[1]}$. Mechanical processing of the refractory brick is also the necessary prerequisites of the preparation of refractories quality inspection sample and thermal equipment building ${ }^{[2]}$, the refractory brick should be machined by the machining equipment to ensure the regular surface of the brick.

There are several problems in the existing equipment:

1. Some of the equipment can't machine the six surfaces of the rectangular brick continuously.

2. Some of the equipment which can achieve processing continuously usually uses sensor and cylinder to change the brick position on the processing line .The cylinder service life is limited, so they can't satisfy high strength continuous processing.

Based on many shortcomings of the existing brick processing equipment, this paper introduces a new type of high efficiency refractory brick processing equipment.

\section{the Overall Design of the Equipment}

The whole structure of the equipment is showed in Fig.(1). The equipment is consisted of some mechanism including Cam turning mechanism, Cutter micro-adjustment mechanism and Steering mechanism of the brick, etc. 


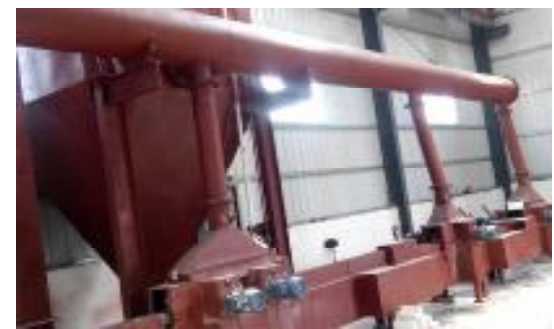

Fig.(1).The whole structure of new
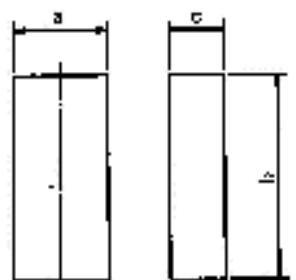

(a)

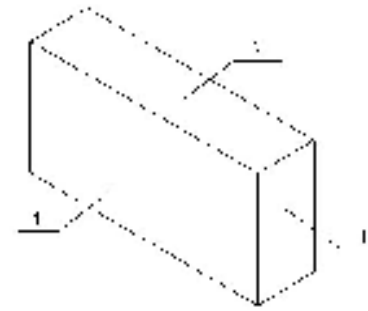

(b)

type refractory brick processing equipment

Fig.(2). Rectangular brick

The rectangular brick is just a straight parallelepiped brick body which is consisted of the length B, width $\mathrm{A}$ and the thickness of the $\mathrm{C}$ three dimensions.

The overall shape of the rectangular brick is showed in Fig.(2)and the processing flow chart of the refractory brick processing equipment is showed in Fig.(3).

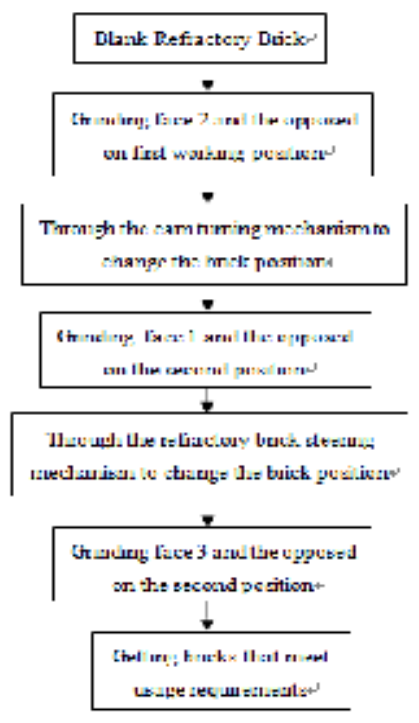

Fig.(3). The processing flow chart of the refractory brick processing equipment

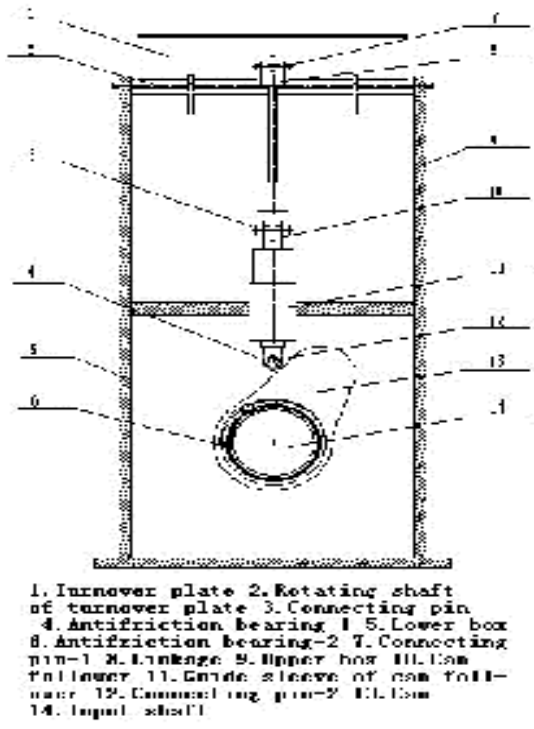

Fig.(4). Interior structure of the cam turning mechanism

\section{The Design of Cam Turning Mechanism}

The cam turning mechanism is a kind of organization that can achieve to change the position of the brick without using sensor and cylinder. Its interior structure is showed in Fig.(5).

The working principle of this mechanism is as follows: The power is input by input shaft. Then, it will drive cam mechanism to make the turnover plate turn refractory bricks over regularly.

\section{The Choice of the Design Plan}

The design of the cam contour line is the key to the mechanism design. There are many kinds of solutions of the CAM contour line, commonly used with analytic method and graphical method. In the same conditions, the graphical method which is based on CAD software is quick, easy and intuitive. The design accuracy of the graphical method design results is as good as the analytic method in the case of dividing fully ${ }^{[3]}$. In this mechanism, the CAM moves in the plane and the steps required cycle reciprocating motion. So the CAM shape is designed for disc CAM based on the above points. According to the requirement of the organization movement, the straight push rod has been used. So which needs to be designed is the contour line of straight moving follower type CAM mechanism. The graphical method of straight moving follower type follower type CAM 
mechanism is introduced in the follows ${ }^{[4]}$.

\section{The Design Principle}

The design method is reversal process. The detail of the reversal process is as follows: When designing cam contour line we can assume that the cam is stationary, the push rod make reverse motion relative to the cam and it move in the guide rail at the same time. We can make a series of positions of the push rod in such a composite movement. Then its trajectory spire is the required cam contour $^{[5]}$.

The design of the cam turning mechanism is based on the cam mechanism. It has several features: 1.Fast response, 2.Simple-compact structure ${ }^{[6]}$, 3.Longer service life, 4.Lower failure rate.

\section{The Design of Cutter Fine Turning Mechanism}

The cutter micro-tensioning mechanism is a kind of organization which can trim cutter in the shaft up. When the cutter is frayed and it is not meet the requirements of machining accuracy. We can trim the position which is in the shaft up of the cutter without changing a new cutter.

The whole structure of the cutter fine tuning mechanism is showed in Fig.(7)and the interior structure of the mechanism is in Fig.(8).

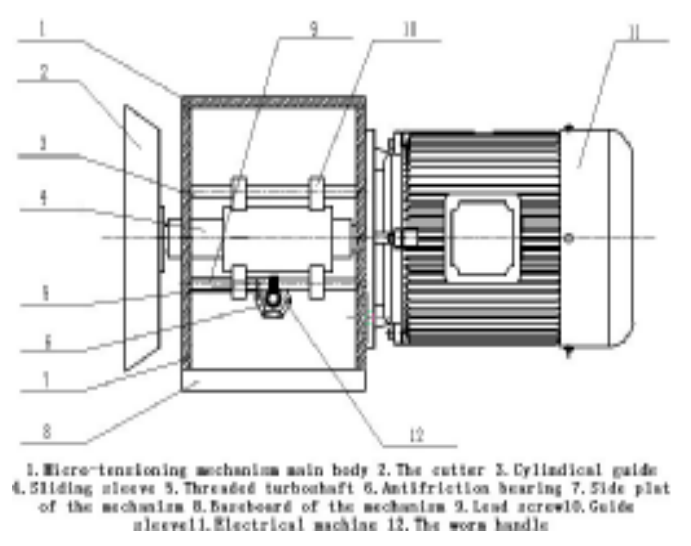

Fig.(5).The whole structure of

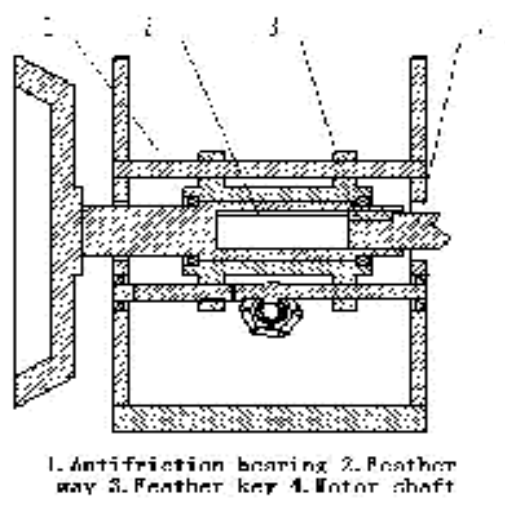

Fig.(6).The interior structure of the mechanism cutter Micro-tensioning mechanism

The working principle of this mechanism is as follows: The cutter is rotating along with the motor shaft by the feather key under normal circumstances. When the cutter is wear and it couldn't meet the machining accuracy, we can rotate the worm handle to adjust the position of the cutter. The rotating of the handle changes into the rotating of the threaded turboshaft by worm gearing. The shaft can only rotate without axial displacement. There is transmission thread at one end of the shaft and we can change the rotation of the shaft into axial displacement of the guide sleeve. The guide sleeve is connected to the sliding sleeve by the bearing interference fit. Finally, we achieve the cutter fine tuning.

The design of the cutter fine tuning mechanism has the following advantages:

1. It is durable, reliable and simplicity of operator; 2 . It can adjust the cutter axial position only by rotating the handle, it makes the production and processing become conveniently and quickly. It also reduces the production costs;3. The threaded connection and the worm drive both are self-locking ensuring the machining accuracy after the adjustment; 4 . The whole structure is in a relatively closed box so it is hard to be affected by dust. The service life has been improved. 


\section{The Design of the Refractory Brick Steering Mechanism}

The refractory brick steering mechanism is a kind of organization that can change the brick by rotating. The discrete function of the mechanism is showed in Fig.(9).The interior structure of the mechanism is showed in Fig.(10).

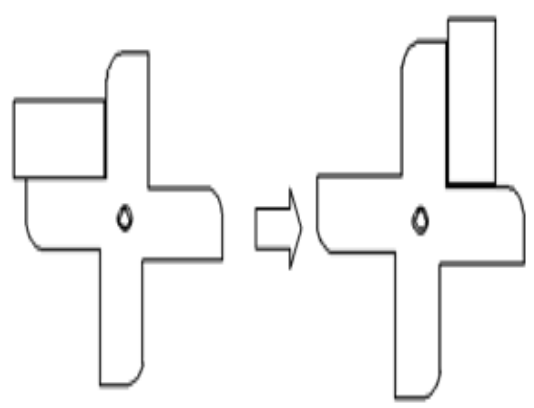

Fig.(7).The function of the refractory brick steering mechanism

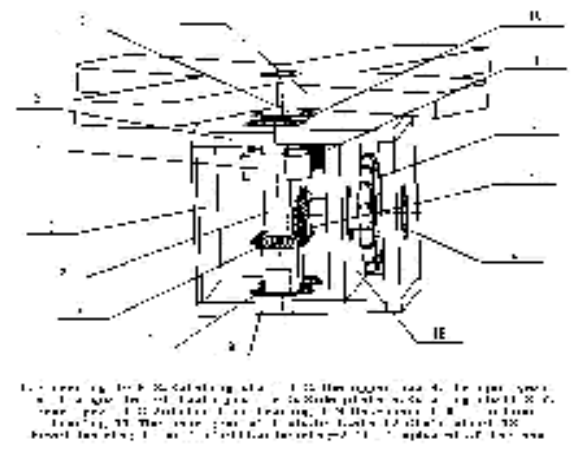

Fig.(8).The interior structure of the mechanism

The working principle of the mechanism is as follows: The chain wheel is the power input to make the rotating shaft- 2 turn by bevel gear drive. The spur gear with a quarter of tooth profile makes the steering fork rotating in the quarter of the chain wheel rotating period become possible.

The advantage of the mechanism is that: 1. It has a simple structure; 2. Using mechanical transmission makes it work reliably; 3.Longer service life; 4.Lower failure rate of the equipment.

\section{Conclusions}

This equipment has been designed based on the service life and reducing the failure rate. We has used some software like SOLIDWORKS and AutoCAD to design the Cam turning mechanism, the cutter micro-tensioning mechanism, the refractory brick steering mechanism to meet the design requirements. Compared with the old rectangular brick processing equipment, it has several advantages:

1. Compared with old brick processing equipment, day processing capacity can be greatly improved. It can machine about 8000 bricks every day.

2. Applying mechanical transmission, Operate stable and safe, producing stable, easy to operate, high degree of automation, Longer service life, Lower failure rate.

\section{References}

[1] Yong-sheng Guo. Development and Application of the New Type Refractory Material Processing Equipment[J] Refractory Material, 1998, 32(3): 183-184

[2] Ping-kun $\mathrm{Xu}$. Significance and Technology Progress of the Refractory Products Machining Operation [J]Refractory Material, 2010, 44(6):470-472

[3] Wan-yun Zhang, Yin-rong Lu, Dong-qing Liang, et al. Graphic and analytical method of designing precision comparison of the cam mechanism[J]. Journal of Light Industry In Chinese, 2011, 155(10):57-58.

[4] Xi-hai Tao. Based on AutoCAD graphical method of mechanism design[J]. Mechanical Research \& Application In Chinese, 2005, 18(6):103-104.

[5] Wen-bo Tang, Chun-yao Wang, Lei Min, Ya-ping Guo. Design of CAM Mechanism for a Kind 
of Chili Directional Pitter [J] Machinery Design \& Manufacture In Chinese,2013,7(7):28-30

[6] Huan Sun, Zuo-mo Chen, Wen -Jie Ge. Mechanical Principles[M]seventh edition. Beijing: Higher Education Press In Chinese, 2006: 151-165

[7] Yong-shun Luo, Hong Tang, Qing-xuan Yang, Xiao-jun Wang. Spherical Motion Design of the RSRR Kneading Mechanism[J]Machinery Design \& Manufacture In Chinese， 2014 ,9(9):84-86 